\title{
CIENTIA
}

\section{COMUNICACIÓN: FACTOR FUNDAMENTAL PARA LA GEGURIDAD AÉREA}

Luz Amparo Laputa Silva*

\section{RESUMEN}

Se presenta la relevancia de la discriminación del lenguaje oral en las tripulaciones aéreas, la importancia de un examen audiológico y la relación de la comunicación oral como factor fundamental en la seguridad de las operaciones aéreas.

Palabras claves: comunicación, seguridad, audición, tripulaciones aéreas, discriminación auditiva.

\section{ABSTRACT}

This article presents the relevance of discriminating oral language in air crews, the importance of a hearing exam, and oral communication as a fundamental factor in the safety of air operations.

"....dos uno seis dos autorizado aproximación pista cero cuatro viento treinta grados ocho nudos $\mathrm{Q} N$ $\mathrm{H}$ dos nueve nueve dos.........,"

Parecería una simple información de las muchas que reciben y envían los pilotos y tripulantes de cualquier aeronave, pero este mensaje puede determinar la seguridad de la operación aérea que se esté realizando, siendo la diferencia entre una misión exitosa o un accidente.

Son múltiples los factores que intervienen en una operación aérea; pero la comunicación, comenzando
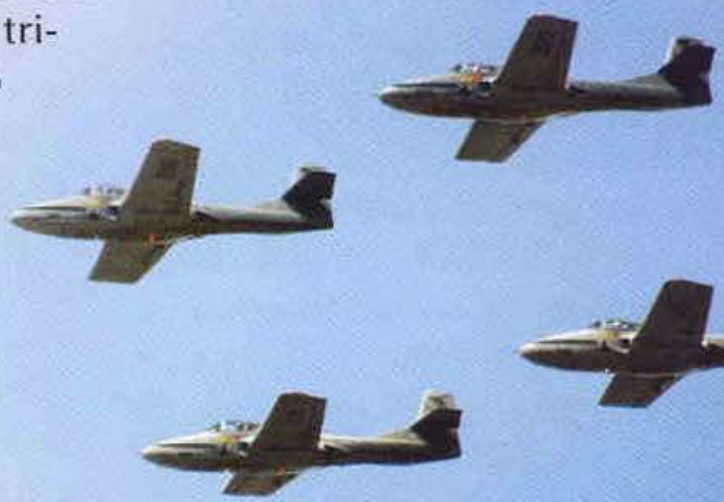
por la capacidad auditiva de cada tripulante, es un aspecto fundamental que conlleva al final de una misión. ¿Por qué la importancia de mantener una buena capacidad auditiva? Toda la información que 


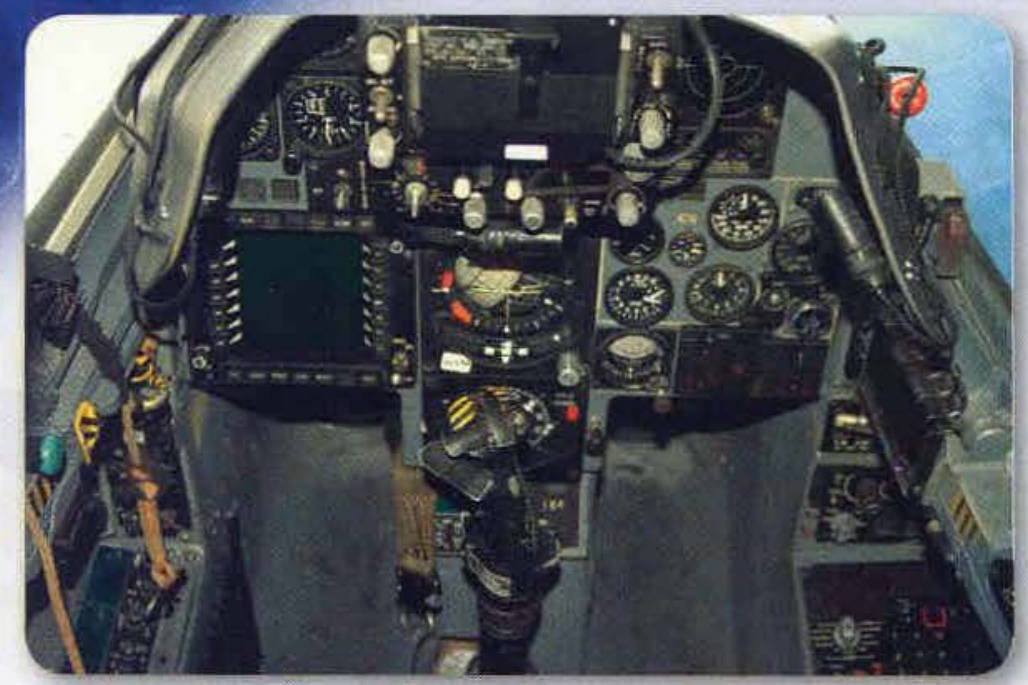

recibimos a nivel oral se obtiene a partir de la capacidad auditiva, la cual se mide en decibeles que se evaluan mediante una serie de pitos o tonos que tienen mayor o menor importancia para la discriminación del lenguaje. Estos tonos se refieren a la frecuencia medida en Hertz. Las frecuencias del lenguaje son 500, 1000 y $2000 \mathrm{~Hz}$. Las demás frecuencias, hasta $8.000 \mathrm{~Hz}$, nos dan otras informaciones adicionales, especialmente acerca de las características de la voz, pero también son similares a pitos utilizados para identificar señales. Muchas de ellas informan acerca de emergencias o condiciones críticas de las aeronaves.

Hemos encontrado que disminuciones auditivas en la zona aguda impiden la discriminación de los sonido/f/, /s/y/t/, los cuales son de baja intensidad pero alta frecuencia, situación muy común en la disminución auditiva inducida por ruido presente en las tripulaciones aéreas. Esto se relaciona directamente con la teoría según la ubicación de los fonemas en el audiograma, al igual que los aspectos suprasegmentales del lenguaje de prosodia y entonación, que son discriminados en forma distorsionada cuando se presentan perdidas auditivas. En vuelo, la intención de un mensaje puede ser interpretado de diferente forma según estas características suprasegmentales, perdiendo así la comunicación. Los requisitos establecidos tanto para la aviación civil como para la militar, exigen que la capacidad auditiva debe estar dentro de los limites 0 a 25 decibeles como máximo para la zona del lenguaje, $y$ hasta 50 decibeles para las frecuencias agudas.

Pero aun más que escuchar los pitos, la mayor importancia está en la discriminación del lenguaje, es decir captar y comprender las indicaciones dadas desde tierra. Por esto a los pilotos se les aplica una prueba de palabras llamada logoaudiometría que mide el porcentaje de discriminación, en la cual deben lograr el ciento por ciento de percepción de las palabras. Esta prueba actualmente es un requisito de la $\mathrm{OACl}$ (Organización de Aviación Civil Internacional) y debe ser con palabras inherentes a la actividad de vuelo.

Además de la capacidad auditiva adecuada para el vuelo, una comunicación adecuada también necesita la competencia comunicativa. A lo largo de la historia, la comunicación ha jugado un papel importante en el desarrollo de la humanidad, aun más en el presente que se podría denominar la era de las comunicaciones. Las relaciones humanas, educativas, laborales, científicas y realmente en general todas necesitan de una comunicación eficaz para que sean armoniosas y saludables. La ciencia y tecnología han puesto al servicio de la comunidad medios y sistemas increíblemente complejos y sofisticados con velocidad y aceleración, como por ejemplo la comunicación establecida entre astronautas y las bases terrestres, la navegación a través de Internet, el contacto entre diferentes pun- 


\section{CIENCIA}

tos del planeta. En contraste con el progreso científico y tecnológico, la comunicación personal, como el intercambio entre las personas en su vida cotidiana, laboral, administrativa y científica, soporta grandes peligros. Esto sucede la mayoría de las veces porque se escucha sin comprender el mensaje ni realizar el intercambio de información, que es la razón de ser de la comunicación.

Desde el punto de vista lingüístico; la comunicación consiste en la transmisión de información, ideas, y mensajes mediante el empleo de signos y palabras. Para el medio aeronáutico, comunicarse es intercambiar información a través de un medio oral (tripulantes-torre) con un propósito especial durante cada una de las etapas del vuelo, y esto implica compartir un mismo lenguaje, lengua $y$ señales.

Es muy importante manejar un mismo lenguaje. Una persona que desconoce el medio aeronáutico no estaría preparado para lograr una adecuada comunicación que permitiera llevar a feliz termino una misión asignada, porque en este lenguaje se encuentra todos los procedimientos y palabras que sin importar la lengua son específicos de cada oficio, profesión o actividad que se realiza.

La lengua (español, inglés, francés, etc.), es el código que permite que esa comunicación sea adecuada. Se apoya en las carac- terísticas paralingüísticas de la lengua como la escritura y la entonación de la voz, y las extralingüísticas que la enriquecen como los símbolos matemáticos y códigos sociales. En el caso de la aviación, el mundo referencial es estar involucrado en la situación de vuelo.

Aún mas importante que todo lo anterior es el mensaje, el cual es el eje de la comunicación. Es una unidad significativa con señales orales que informan (en el caso de la aviación) acerca de una situación o procedimiento a realizar. Existen mensajes buenos que cubren el interés y las expectativas, la necesidad o carácter de lo que se busca; y los mensajes vacíos que no llevan la información NECESARIA. Este punto muy importante de tener en cuenta en situaciones de emergencias aéreas, porque puede haber información pero no completa el requerimiento de ser mensaje bueno.

El canal verbal-oral cumple la función más importante en las comunicaciones a nivel del medio aeronáutico. Según Kaplun (1998), la verdadera comunicación no comienza hablando sino escuchando. Es necesario ser conciente de para qué se comunica y qué se pretende con la comunicación. La importancia de la retroalimentación está en preguntarse inter- 
namente si la otra persona se interesa por el mensaje enviado, entiende, lo sigue, algo anda mal, por qué pregunta o afirma, etc. Esta competencia comunicativa solo se logra con una adecuada retroalimentación. En una cadena comunicativa es fundamental estar atento, "estar alerta" a cada situación o mensaje, porque esto permitirá reorientar o ajustar la información posterior.

Las fallas en la comunicación es uno de los factores humanos que se presenta con mayor frecuencia, aumentando la cadena del error durante la ventana de riesgo, pues toda la información recibida y enviada por el equipo de trabajo dentro de la aeronave debe buscar mantener la conciencia situacional en la cabina. Según una investigación realizada por Wiener y Nagel; el 10 $\%$ de los accidentes la tripulación ignora información externa y el $6 \%$ presenta una comunicación deficiente con el controlador aéreo ubicado en la torre. Estas fallas, principalmente de comunicación, ocurren por omitir o malinterpretar la información.

Algunos accidentes reportados por factor comunicación son los siguientes.

- En Tenerife, Islas Canarias, en 1977, dos aeronaves $\mathrm{B}-747$ colisionaron por fallas en la comunicación. El mensaje fue entrecortado y no supieron a quién iba dirigido, se afectó la trasmisión de la frecuencia, no se aseguraron de confirmar el mensaje y la torre no percibió el error en la recepción del mensaje.

- En Milán el 08 de octubre de 2001, ocurrió un caso de incursión en pista de otra aeronave y la posterior colisión entre un avión MD-87 de Scandinavian Airlines System y un Cessna Citation 525, luego de que en condiciones de baja visibilidad se interpretó mal una línea amarilla y no se expresaron las dudas entre la tripulación.

- Otra colisión sucedió entre un B-737 y un Metro II en Los Ángeles, luego que la controladora sufriera de un error en la comunicación que no pudo solucionar, dejando el Metro en la cabecera esperando autorización de despegue mientras el B-737 estaba en final en la pista.

- El 26 de Marzo de 2001 dos F-15 de la Fuerza Aérea de Estados Unidos (USAF) colisionaron con el terreno en una tormenta de nieve cuando solicitaron una altura mínima de vuelo empleando el término americano que el controlador de radar británico no entendió. El creyó que los pilotos veían el piso y podían volar visual, cuando en realidad estaban solicitando vectores.

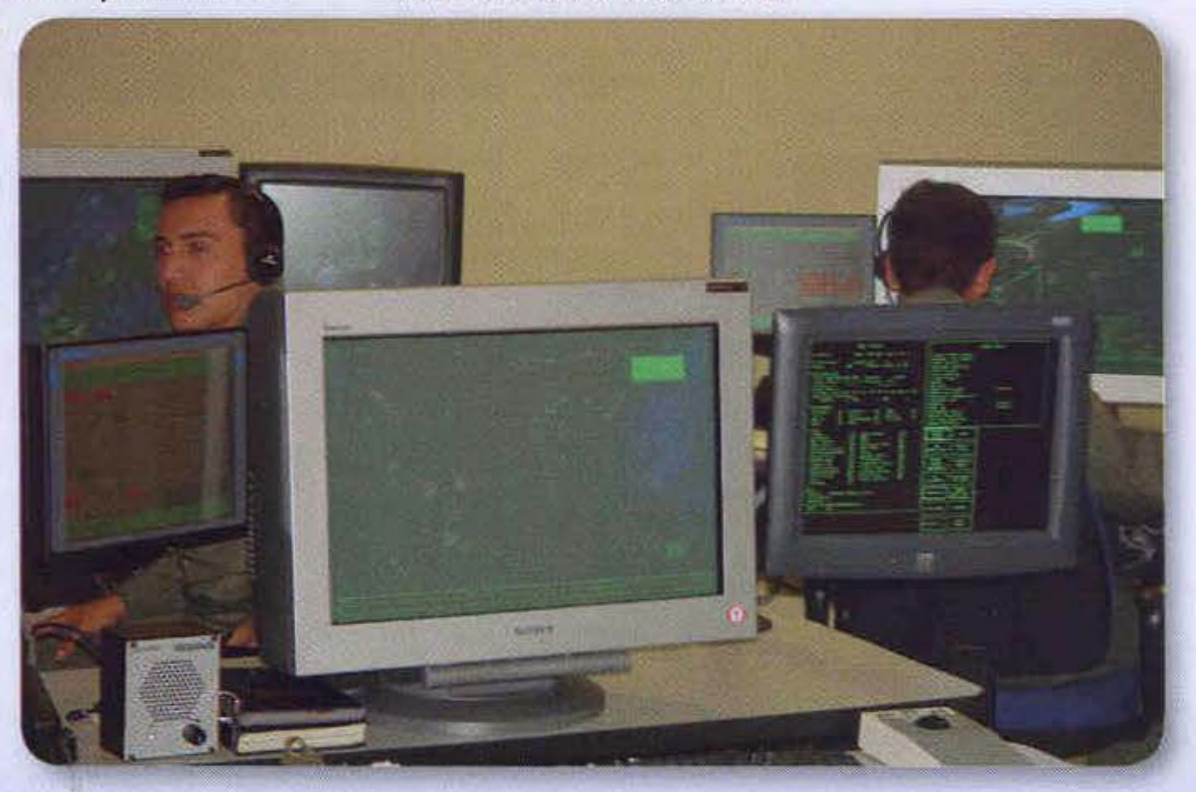


- Un caso Colombiano es el del Avianca 052 hacia Nueva York el 25 de enero de 1990, cuando la tripulación no pudo evidenciar a la torre sus necesidades reales o condición de emergencia por la falta de combustible, y el aparato se estrelló contra una zona residencial a la orilla norte de Long Island.

Al analizar los accidentes, las fallas en comunicación son consideradas como pasivas, pero iqué tan pasiva puede ser la comunicación si de ella depende todo el proceso desde que se ubica el tripulante en la cabina hasta que finaliza en otro lugar? Toda la relación que se mantenga con la tierra necesita de la comunicación para lograr la misión. La capacidad auditiva y la competencia comunicativa, entonces, son dos factores muy importantes porque permiten una correcta comunicación oral, entendida esta como el intercambio adecuado de mensajes que permita mantener la seguridad de las operaciones aéreas y disminuir los accidentes.

\section{BIBLIDERAVIA}

1. NINO, Victor Miguel. Competencia en la comunicación. Editorial Ecoe, Colombia, 2003.

2. Aeromedical Educafion (Medical Facts for Pilots)

3. REINHART, Richard O., M.D. Basic Flight Physiology

4. GRACIA, Orlando. M.D., Seminario sobre "Factores Humanos". Colombia, 2000.

5. Departamento de Seguridad, Inspección General Fuerza Aérea Colombiana. "Reducción de los accidentes en la aproximación y. el despegue, 2003

6. Avianca. "Curso de factores humanos": 2804

7. AIFSSANDRO, Martin. Genetica Della funzione uditiva normale e patologica. Omega Edizione, Torino, Itahia, 2006.

8. LUIS, Felipe Sexto. "El ruido: enemigo público numero uno:" Cuba, 2002.

9. Departamento de Seguridad Aérea e industrial. Inspección General, Fuerza Aérea Colombiana. "Entrenamiento para la reducción del error humano en la aviación". Colombia, 2003.

10. CT Juan Felipe Rodriguez Arana, MY Alejandro Torres Cogollo. Colaboración y restimonio.

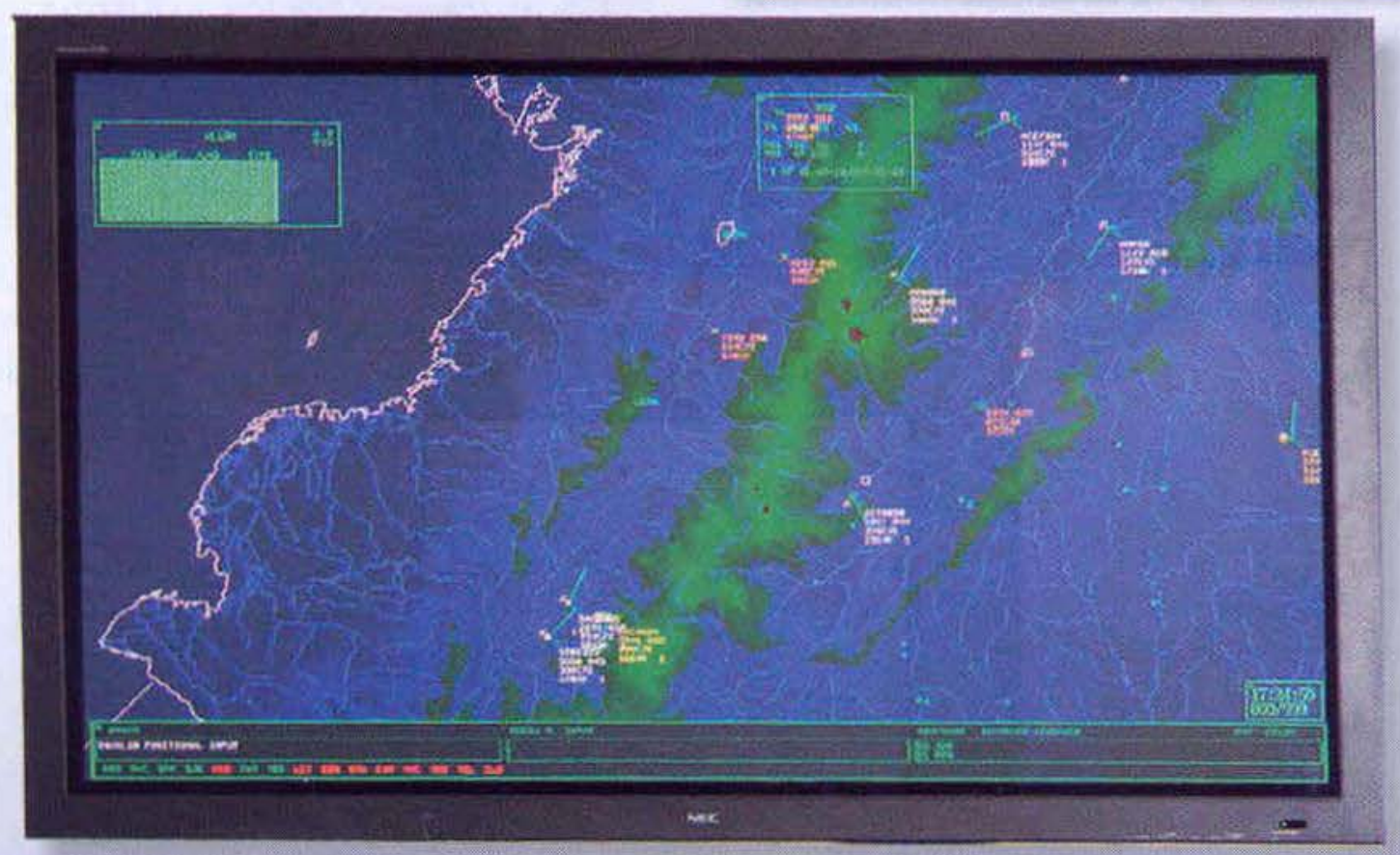

